\section{Anonyme \\ Entreprises et développement économique local \\ Paris, La Documentation française, DATAR collection Territoires en mouvement 2004, 103 pages}

Souhaitons qu'avec sa nouvelle identité, l'ex DATAR, devenue la DIACT (Délégation interministérielle d'aménagement et de compétitivité des territoires) maintiendra cette collection ( «Territoires en mouvement ») qui nous a fait bénéficier, entre autres, de titres tels que Les pays; La prospective territoriale; La Montagne; Les système productifs locaux (ce dernier fut recensé dans ces pages). Une fois n'est pas coutume, l'auteur du présent ouvrage a préféré conserver l'anonymat. Il est vrai qu'il s'agit avant tout d'une sorte de vade macum du développement économique local (DEL) : tout ce qu'on devrait savoir sur le sujet sans toujours oser le demander. Sans forcément prétendre à l'exhaustivité, tout en présentant des exemples riches en information, l'ouvrage attire l'attention sur les raisons qui amènent les entreprises à s'engager en faveur du développement local (DL) et sur les conditions de réussite de ces démarches.

Les processus et dispositifs visant l'emploi se trouvent donc, ici, bien mis en évidence. Et puisqu'il est question de DL, on se rapporte en conséquence aux actions très diverses mises de l'avant par des acteurs locaux. Mais pour s'assurer de bien comprendre ce dont il est effectivement question en se référant à une démarche de DL, le lecteur a intérêt à consulter l'annexe dès l'ouverture du petit volume. Car, on le sait, nos cousins français ont parfois tendance à galvauder l'expression « développement local » en abusant du fait qu'une activité, par définition, doit bien se localiser quelques part... Alors, on peut lire qu'avec le DL, on se rapporte à toute intervention dont l'initiative revient à des acteurs exerçant des responsabilités locales, qui a un effet visible et évaluable sur des champs tels que :

- le développement économique et, par voie de conséquence, la création d'emplois;

- l'amélioration des systèmes locaux de formation initiale ou continue;

- les initiatives culturelles;
- l'appui à l'insertion de populations en voie d'exclusion ou fragiles (le social qui s'allie à l'économique, $\mathrm{N}$ de l'A).

Et l'on souligne que le DL est souvent associé à l'idée de démocratie locale. On ne peut se voir plus en accord.

Fort de ces prémisses, le lecteur peut mieux saisir la portée des premières lignes du chapitre 1 où il est précisé que l'ouverture de la grande entreprise sur son environnement territorial - sa participation au développement du territoire où elle est implantée - est, en France, un phénomène assez récent dont l'apparition se situe vers la fin des années 1970. Et au Québec alors ?, direz-vous. On attend toujours. L'Alcan à Saguenay, la Kruger à Trois-Rivières? Allons! Un des rares exemples que j'ai connus, c'est il y a 20 ans : un géant de l'alimentation qui a offert son appui à un incubateur dans l'ouest de Montréal en suivant l'exemple de British Steel avec son programme $J o b$ Creation, au début des années 1980.

Mais revenons en Hexagone à la faveur de l'exemple de Saint-Gobain Développement créé en 1979 et découvert à l'occasion d'un de mes voyages d'études (où j'ai toujours su joindre l'utile à l'agréable). Considéré comme le principal outil de la politique du groupe Saint-Gobain à l'égard des territoires, l'organisme a, parmi ses objectifs, celui de participer au DL par des actions de soutien aux PME de différents bassins d'emploi où il est implanté. Dans un chapitre intitulé « Les actions visant à développer l'esprit entrepreneurial et la création d'entreprises », il est fait allusion à d'autres organismes qu'il m'a été donné de connaître lors de mes pérégrinations au pays des ancêtres et dont je suis heureux d'apprendre qu'apparemment, ils sont toujours en existence. Ce sont, par exemple, France initiative réseau qui fédère pas moins de 250 plates-formes d'initiatives locales ayant pour fonction d'aider les porteurs de projet à créer leur entreprise. Ou encore Réseau Entreprendre, créé par un dirigeant d'une grande entreprise qui a su attirer d'autres dirigeants dans son sillon. Ainsi, vingt ans après sa création elles sont une trentaine d'associations dans l'ensemble du pays à assurer une aide personnalisée, régulière et gratuite au futur créateur d'entreprise. Pour ne pas allonger indûment la litanie, je mentionne pour terminer l'essaimage favorisé par France Télécom. Un réseau de délégués à l'essaimage a été mis en place, avec pour objectif d'accompagner les salariés porteurs 
de projet de création. Des résultats : 455 salariés ont créé 350 entreprises qui ont généré 1940 emplois (TPE ${ }^{1}$, il va sans dire : tite, tite, tite, oui, mais c'est mieux que le $\mathrm{BS})^{2}$. Un exemple : reprise d'une auberge à côté du tunnel trans-Manche. Avouez-le! Comme moi, vous aurez envie de vous y arrêter la prochaine fois.

Enfin, je recommande ce modeste ouvrage également pour son glossaire et les contacts (sites Web précisés) qui y sont fournis en annexe.

Note

1 TPE signifie « très petite entreprise ».

2 BS fait référence au «Bien-être social » qui est l'appellation populaire pour le programme d'assistance-emploi (autrefois appelé aide sociale) du ministère de l'Emploi et de la Solidarité sociale du Québec.

\section{Robert Marconis \\ France : recompositions territoriales Paris, La Documentation française coll. Documentation photographique 2006, 64 p.}

Un numéro spécial, format $O \& T$ en en papier glacé, comprenant de magnifiques cartes et de non moins superbes photos toutes en couleurs (avec en couverture une photo du fameux viaduc de Millau qui, lui, ne se rendra pas célèbre en s'effondrant...). Vous voulez tout savoir sur l'histoire de la régionalisation en France et sur la façon dont nos cousins vivent la décentralisation? Vous aurez réponse à vos interrogations avec ce numéro dû à un collègue géographe de l'Université Toulouse-le-Mirail. Régions, départements, communes (toujours au nombre de 36500 ), communautés de communes, "pays », SCOT, pôles de compétitivité, parcs nationaux, etc. Tout ceci se trouve offert à travers la description d'un long processus marqué par la déconcentration, la décentralisation et la régionalisation.

Plus de pouvoir aux régions : voilà un thème qui touche droit au cœur les gens de nos régions dites périphériques. Ces derniers seront intéressés d'apprendre que, durant les années 1960, les régions administratives de l'Hexagone se situaient sous la dépendance d'un État centralisé, conformément à l'étymologie du mot région : regere qui veut dire dominer, régner diriger... Ouf! Avec les lois de 1982, comme le montre très bien ce dossier, bien des choses ont changé et nos idylles régionales peuvent y trouver une source d'inspiration. Alors qu'au Québec on fusionne et défusionne sans scrupule, ici on voit comment la fille aînée de l'Église met en œuvre des regroupements intercommunaux susceptibles de développer des projets solidaires.

\section{André Joyal}

Université du Québec à Trois-Rivières 


\section{Publicité}

\section{Site Web ROT}

